\title{
In vivo assessment of antiretroviral therapy-associated side effects
}

\author{
Eduardo Milton Ramos-Sanchez ${ }^{1,2}$, Hiro Goto ${ }^{2,3}$, Dolores Helena Rodriguez Ferreira Rivero ${ }^{4}$, \\ Thais Mauad ${ }^{4}$, Fernando Nogueira de Souza ${ }^{5}$, Andrea Moreira Monteiro', Magnus Gidlund ${ }^{1 /+}$ \\ 'Departamento de Imunologia, Instituto de Ciências Biomédicas ${ }^{2}$ Instituto de Medicina Tropical \\ ${ }^{3}$ Departamento de Medicina Preventiva ${ }^{4}$ Departamento de Patologia, Faculdade de Medicina \\ ${ }^{5}$ Departamento de Clínica Médica, Faculdade de Medicina Veterinária e Zootecnia, Universidade de São Paulo, São Paulo, SP, Brasil
}

Antiretroviral therapy has been associated with side effects, either from the drug itself or in conjunction with the effects of human immunodeficiency virus infection. Here, we evaluated the side effects of the protease inhibitor (PI) indinavir in hamsters consuming a normal or high-fat diet. Indinavir treatment increased the hamster death rate and resulted in an increase in triglyceride, cholesterol and glucose serum levels and a reduction in anti-oxLDL autoantibodies. The treatment led to histopathological alterations of the kidney and the heart. These results suggest that hamsters are an interesting model for the study of the side effects of antiretroviral drugs, such as PIs.

Key words: metabolic disorders - protease inhibitor - HIV

Indinavir is a potent protease inhibitor (PI) that has been used in adults and children in combination with nucleotide reverse transcriptase inhibitors to control the progression of human immunodeficiency virus (HIV) infection. This drug is currently still used in patients who initiated their therapy with this PI and maintains a low viral load due to its high penetration into viral reservoirs (Bertrand et al. 2009).

The continuous use of antiretroviral therapy (ART) has been associated with a number of undesirable side effects that are associated with a range of metabolic complications and include dyslipidaemia, insulin resistance, overt diabetes mellitus and sudden death (Carr et al. 1998, Anastos et al. 2007, George et al. 2010). These side effects may be a result of the drug itself or of combination with the effects of HIV infection.

Thus, the ability to directly evaluate the side effects of antiretroviral drugs in a non-human model is of upmost importance. Considering the great similarities in plasma lipid composition and lipid metabolism between humans and hamsters, we postulated that hamsters (Mesocricetus auratus) might be an appropriate proxy for evaluation of the side effects of antiretroviral drugs (Alexaki et al. 2004).

Side effects are one of the major reasons for non-adherence to ART, the rate of which reaches $25 \%$ in Brazil. Therefore, we urgently need models through which side effects can be evaluated before the approval of drugs for clinical use. The present study aimed to evaluate the side effects of the PI indinavir in hamsters, as a proxy, by

doi: $10.1590 / 0074-0276130559$

Financial support: CAPES (fellowship to EMR-S), INCT-INAMI/CNPq + Corresponding author: gidlundm@usp.br

Received 2 December 2013

Accepted 8 April 2014 analysing the lipid profile and glucose levels, the levels of anti-oxidised low-density lipoprotein (oxLDL) autoantibodies as a marker of the atherosclerotic process and renal and cardiac histopathological alterations in hamsters given a normal or high-fat diet.

Forty-eight hamsters between 40-60 days old were assigned to one of four study conditions: (i) a normal diet without treatment, (ii) a normal diet and indinavir treatment, (iii) a high-fat diet without treatment and (iv) a high-fat diet and indinavir treatment. The high-fat diet consisted of $20 \%$ saturated fat from coconuts and $0.4 \%$ cholesterol (Sigma-Aldrich, USA) (Alexaki et al. 2004). To establish an optimal dosage, 3, 6, 12 and $30 \mathrm{mg} / \mathrm{kg} /$ day of indinavir were tested and the last dosage was chosen for further evaluation.

All animals were followed for survival analysis and 10 randomly selected animals from each group were evaluated based on selected parameters. The survival analysis was conducted using the Kaplan-Meier survival curve (MedCalc statistical software, Belgium). At 90 days, following 16-18 $\mathrm{h}$ of fasting, blood samples were collected from the hamsters, which were anaesthetised using 200 $\mathrm{mg} / \mathrm{kg}$ ketamine and $10 \mathrm{mg} / \mathrm{kg}$ intraperitoneal xylazine and then sacrificed in a $\mathrm{CO}_{2}$ chamber. Tissues were then removed for histopathological analysis. The Gaussian distribution was confirmed by the Shapiro-Wilk test and the differences were analysed using ANOVA and TukeyKramer tests via GraphPad Prism 5.0 software ${ }^{\circledR}$ (GraphPad Software Inc, USA). The level of significance was set at $p \leq 0.05$. The study was approved by Bioethical Committee for Experimental Animals of the institution.

Initially, 3, 6 and $12 \mathrm{mg} / \mathrm{kg} / \mathrm{day}$ of indinavir were tested for six months in animals given the normal or highfat diet. At $3 \mathrm{mg} / \mathrm{kg} / \mathrm{day}$, no alterations were observed in any of the evaluated parameters. At $6 \mathrm{mg} / \mathrm{kg} / \mathrm{day}$, we observed higher cholesterol and triacylglycerol (TG) levels at two months of treatment compared with levels in untreated animals in the high-fat diet group. At $12 \mathrm{mg} / \mathrm{kg} /$ day of indinavir, higher TG levels were observed in the first month of treatment in treated animals compared with 
untreated animals in the high-fat diet group. An increase in cholesterol levels was also observed after three and four months of treatment in animals receiving the normal or high-fat diet compared with the respective groups of untreated animals. At this dosage, the first deaths occurred at approximately 60 days. Survival rates were lower in animals treated with $12 \mathrm{mg} / \mathrm{kg} /$ day of indinavir and fed the high-fat diet $(50 \%, \mathrm{p} \leq 0.0001)$ and in the treatment and normal diet groups $(82.5 \%)$ compared with the untreated control group $(100 \%)$ at 180 days of treatment. Proceeding with $30 \mathrm{mg} / \mathrm{kg} /$ day of indinavir, the survival rate was lower in the treated animals given the high-fat $\operatorname{diet}(25 \% ; \mathrm{p}=0.0008)$ and in those treated and given the normal diet (32.5\%) compared with the untreated control group $(92.5 \%)$ at 120 days of treatment. Because the lower dosages took a longer amount of time to induce overt side effects, we chose the dosage of $30 \mathrm{mg} / \mathrm{kg} / \mathrm{day}$ for the purpose of analysing the chosen parameters.

Irrespective of the type of diet, indinavir treatment resulted in a dramatic reduction of lifespan. With the introduction of ART for the treatment of HIV infection, there was an increase in cardiovascular complications, several of which have been attributed to atherosclerosis (George et al. 2010, Ronchini et al. 2013). Additionally, certain studies have shown that HIV PIs cause a prolongation of ventricular repolarisation that may induce various cardiac arrhythmias, such as polymorphic ventricular tachycardia, leading to syncope and even sudden death in human subjects (Singh et al. 2010). Sudden death due to indinavir-induced nephropathy has also been reported (Ras et al. 2000). No consensus exists on whether HIV PIs lead to sudden death, although high cumulative exposure to PIs has been associated with this outcome (Worm et al. 2012). As a result, cardiac and renal histological alterations were a focus of the present study.

To locate atherosclerotic lesions, the aortic arch was removed and segments of the heart and aortic arch were serially sectioned ( $5 \mu \mathrm{m}$ thick). The histological sections $(3-\mu \mathrm{m})$ of the heart and kidney were stained with haematoxylin-eosin (H\&E), Masson's trichrome, periodic acid methenamine (PAM) and Oil Red $\mathrm{O}$ dyes and analysed using light microscopy.

The histological evaluation of heart tissues stained with Masson's trichrome dye showed areas of fibrosis in animals treated with indinavir under both the normal and the high-fat diet conditions (B, D in Figure) compared with untreated control animals (A, C in Figure). No lesions suggestive of atherosclerosis were found in either the aortas or the carotid arteries in the samples stained with H\&E or Oil Red O.

In the kidneys stained with $H \& E$ dye, we observed hyperplasia of the podocytes and a decrease in the Bowman's capsule space (data not shown) in the animals treated with indinavir, regardless of the type of diet. When Masson's trichrome dye was used, a decrease in the Bowman's capsule space and in areas of fibrosis was found in the groups treated with indinavir (F, H in Figure) compared with the two control groups (E, G in Figure). The histological sections of kidney stained with PAM dye demonstrated capillary wall thickening and a reduction in the Bowman's capsule space in both of the indinavir treatment groups (data not shown). No histological alterations of the kidney were observed in the untreated control groups.

Among PIs, indinavir is the inhibitor most frequently associated with kidney alterations. In the present study, we observed interstitial fibrosis of the kidney, hyperplasia of podocytes and a decrease in the Bowman's capsule space, which are side effects similar to those reported in humans undergoing indinavir treatment. However, none of the evident functional changes reported in patients under indinavir treatment (Boubaker et al. 1998, Daugas et al. 2005) were present. One evident histopathological alteration in both the kidneys and the heart was the presence of areas of fibrosis. This phenomenon may have been a result of the high indinavir dosage used, but the premature death, similar to that observed in humans undergoing ART, led us to carefully consider this finding as the putative cause of death that may have led to dysrhythmia. Another important finding in the present study was the absence of atherosclerotic lesions in the heart vasculature, suggesting no evident role for ischemic episodes in death during ART. We believe that the absence of marked atherosclerotic lesions may have been due to the length of our experiment, which was shorter than the time span necessary for the development of atherosclerosis in hamsters (approximately 9 months) (Ugawa et al. 2002). Therefore, we explored an alternate parameter, which was not detected histopathologically, to understand the on-going atherosclerotic process.

To evaluate biochemical parameters, serum urea, creatinine, aspartate transaminase and alanine transaminase (ALT), plasma glucose, TG, total cholesterol (TC) and high-density lipoprotein (HDL) levels were determined using a commercial kit (Reflotron, Roche, Switzerland) according to the manufacturer's protocol. The LDL concentration was estimated using the Friedewald equation (Anastos et al. 2007) and the serum auto-antibodies against oxLDL were measured by ELISA, as previously described (Soares et al. 2009).

A significant increase in triglyceride, cholesterol and glucose levels in animals after 90 days of treatment with indinavir was observed in both the normal and the highfat diet groups compared with the respective untreated control groups. No significant differences in HDL, LDL, urea or creatinine levels or ALT activity were observed (Table). No significant differences among groups with respect to these parameters were encountered at the outset of the experiment (data not shown).

Anti-oxLDL levels were lower in the indinavir-treated animals, irrespective of whether they received the normal $(0.19 \pm 0.05 ; \mathrm{p}=0.0004)$ or high-fat $(0.21 \pm 0.06 ; \mathrm{p}=$ $0.0004)$ diet, compared with untreated animals given the normal $(0.30 \pm 0.11)$ or high-fat $(0.33 \pm 0.07)$ diet.

A large number of clinical studies have shown that the presence of antibodies against modified lipoproteins (e.g., oxLDL) is a marker for atherosclerosis in several pathologies, including HIV (Cunha et al. 2013, Ronchini et al. 2013). The exact role of these antibodies remains unclear, but the importance of these antibodies in either aggravating or protecting against atherosclerosis is evident. These studies suggest that the decrease in anti-oxLDL levels is 
related to the generation of oxidised LDL, which may induce the atherosclerotic process. In this context, the decrease in anti-oxLDL levels observed in the present study may suggest an on-going atherosclerotic process in animals under indinavir treatment. Importantly, the results suggest that the decreased levels of anti-oxLDL are directly related to the treatment and not to the type of diet.
With indinavir treatment, several biochemical alterations observed in humans were also observed in hamsters, such as an increase in glucose, triglyceride and TC levels. In the present study, no significant difference in HDL and LDL levels was observed, although slight or moderate increases in HDL and LDL serum levels have been reported in relation to certain ART therapies, but
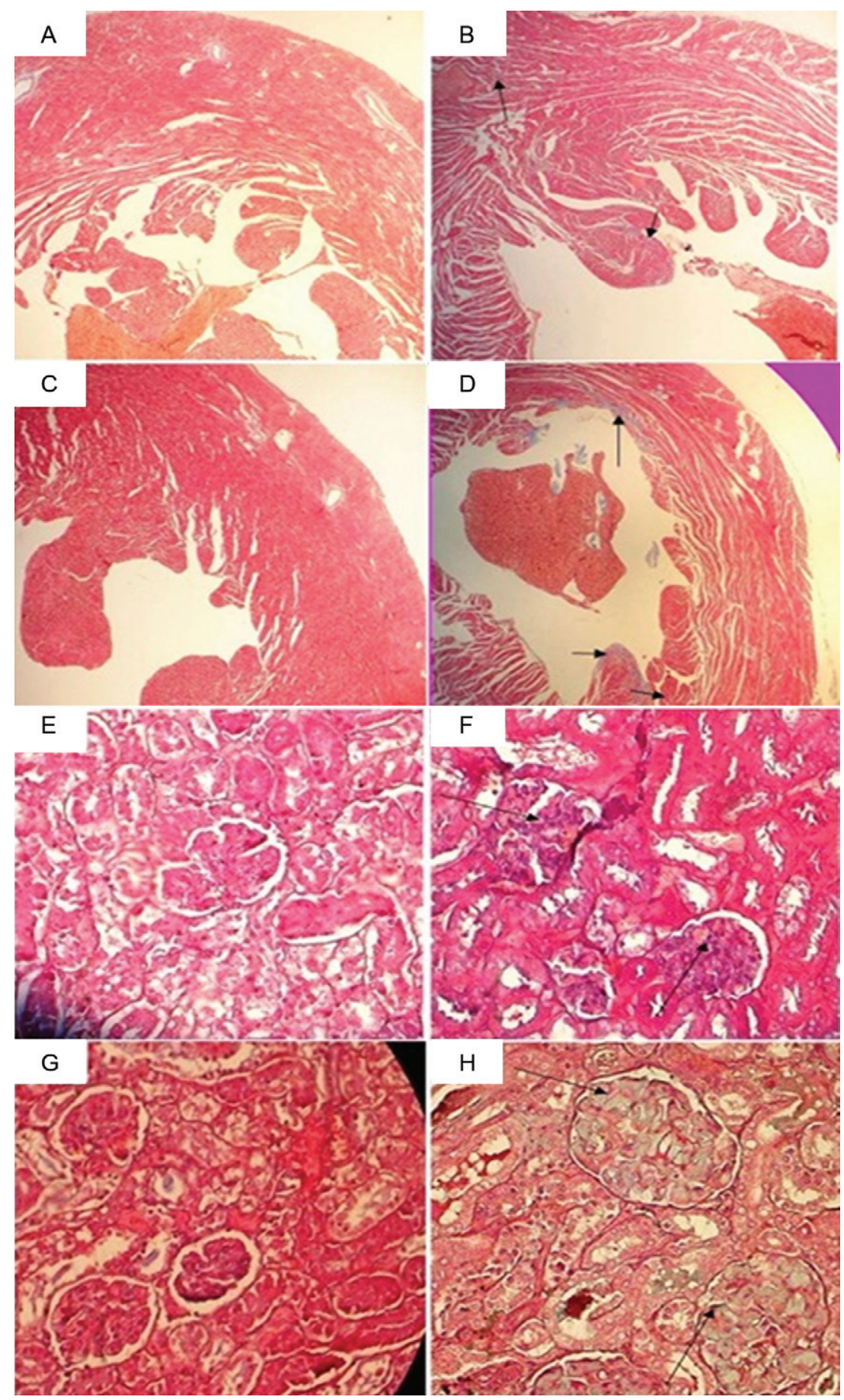

Histological evaluation of heart and kidney tissues of Mesocricetus auratus under indinavir treatment with or without high fat-diet. Hamster heart (A-D) and kidney (E-H) histological sections stained with Masson's trichrome dye (40X). Areas of fibrosis stained in blue are indicated by arrows. A, E: normal diet without treatment; B, F: normal diet with $30 \mathrm{mg} / \mathrm{kg} / \mathrm{day}$ indinavir treatment; C, G: high-fat diet without treatment; D, $\mathrm{H}$ : high-fat diet with $30 \mathrm{mg} / \mathrm{kg} /$ day indinavir treatment. 


\section{TABLE}

Triacylglycerol (TG), total cholesterol (TC), high-density lipoprotein (HDL), low-density lipoprotein (LDL), glucose, urea, creatinine, aspartate transaminase (AST) and alanine transaminase (ALT) levels in blood serum at 90 days of treatment

\begin{tabular}{lcccc}
\hline & \multicolumn{3}{c}{ Groups } \\
\cline { 2 - 5 } Parameters & Normal diet & $\begin{array}{c}\text { Normal diet } \\
+ \text { indinavir }\end{array}$ & High-fat diet & $\begin{array}{c}\text { High-fat diet } \\
+ \text { indinavir }\end{array}$ \\
\hline TG (mg/dL) & $160.0 \pm 14.9$ & $233.0 \pm 55.3^{a}$ & $272.1 \pm 35.9^{b}$ & $351.7 \pm 83.6^{a, b}$ \\
TC (mg/dL) & $88.1 \pm 17.3$ & $99.6 \pm 14.5$ & $257.2^{c} \pm 32.2^{c}$ & $318.0 \pm 60.2^{a, c}$ \\
HDL (mg/dL) & $45.4 \pm 13.3$ & $51.2 \pm 5.5$ & $88.9 \pm 13.5^{c}$ & $106.7 \pm 26.0^{c}$ \\
LDL (mg/dL) & $17.6 \pm 15.6$ & $16.6 \pm 6.7$ & $119.3 \pm 25.1^{c}$ & $127.2 \pm 24.8^{c}$ \\
Glucose (mg/dL) & $77.7 \pm 6.2$ & $92.9 \pm 8.7^{a}$ & $90.9 \pm 9.7$ & $107.5 \pm 16.6^{a, b}$ \\
Urea (mg/dL) & $46.3 \pm 8.6$ & $46.7 \pm 4.2$ & $29.0 \pm 7.4^{c}$ & $30.6 \pm 13.4^{c}$ \\
Creatinine (mg/dL) & $0.25 \pm 0.05$ & $0.26 \pm 0.02$ & $0.32 \pm 0.03^{b}$ & $0.28 \pm 0.07$ \\
AST (U/L) & $15.5 \pm 5.8$ & $30.3 \pm 9.1^{a}$ & $7.1 \pm 7.8$ & $32.4 \pm 7.9^{a}$ \\
ALT (U/L) & $35.4 \pm 13.0$ & $49.9 \pm 5.6$ & $57.9 \pm 18.2^{b}$ & $54.1 \pm 19.3$ \\
\hline
\end{tabular}

$a: \mathrm{p} \leq 0.05$ in relation to their respective untreated controls; $b: \mathrm{p} \leq 0.05$ in relation to the untreated control under normal diet; $c: \mathrm{p} \leq 0.05$ in relation to treated and untreated animals under normal diet. The results are the means \pm standard deviation. Superscripts indicate $\mathrm{p} \leq 0.05$ (ANOVA and Tukey-Kramer tests). Indinavir treatment ( $30 \mathrm{mg} / \mathrm{kg} / \mathrm{day}$ ).

not specifically with indinavir (Calza et al. 2008, Ronchini et al. 2013). Thus, if an atherosclerotic process is occurring, it is more strongly related to modifications of LDL particles, as observed by the measurement of antioxLDL levels, than to changes in LDL levels.

The present study demonstrated that $M$. auratus could be used to closely reproduce toxic drug side effects observed in humans. This study showed an outcome similar to the occurrence of sudden death observed in patients undergoing ART and we also observed biochemical, pathological and immune markers that are appropriate for physiopathological analysis and may be related to this outcome. Thus, this novel model should be considered for the future evaluation of candidate antiretroviral drugs.

\section{REFERENCES}

Alexaki A, Wilson TA, Atallah MT, Handelman G, Nicolosi RJ 2004. Hamsters fed diets high in satured fat have increased cholesterol accumulation and cytokine production in the aortic arch compared to cholesterol-fed hamsters with moderately elevated plasma non-HDL cholesterol concentrations. J Nutr 134: 410-415.

Anastos K, Lu D, Shi Q, Tien PC, Kaplan RC, Hessol NA, Cole S, Vigen C, Cohen M, Young M, Justman J 2007. Association of serum lipid levels with HIV serostatus, specific antiretroviral agents and treatment regimens. J Acquir Immune Defic Syndr 45: 34-42.

Bertrand J, Treluyer J-M, Panhard X, Tran A, Auleley S, Rey E, Salmon-Céron D, Duval X, Mentré F 2009. Influence of pharmacogenetics on indinavir disposition and short-term response in HIV patients initiating HAART. Eur J Clin Pharmacol 65: 667-678.

Boubaker K, Sudre P, Bally F, Vogel G, Meuwly J-Y, Glauser MP, Telenti A 1998. Changes in renal function associated with indinavir. AIDS 12: F249-F254.

Calza L, Manfredi R, Pocaterra D, Chiodo F 2008. Risk of premature atherosclerosis and ischemic heart disease associated with HIV infection and antiretroviral therapy. $J$ Infect 57: 16-32.
Carr A, Samraas K, Chisolm DJ, Cooper DA 1998. Pathogenesis of HIV-1 protease inhibitor-associated peripheral lipodistrophy, hyperlipidemia and insulin resistance. Lancet 351: 1881-1883.

Cunha J, Maselli LMF, Treitinger A, Monteiro AM, Gidlund M, Maranhão RC, Spada C, Bydlowski SP 2013. Serum levels of IgG antibodies against oxidized LDL and atherogenic indices in HIV1-infected patients treated with protease inhibitors. Clin Chem Lab Med 51: 371-378.

Daugas E, Rougier JP, Hill G 2005. HAART-related nephropathies in HIV-infected patients. Kidney Int 67: 393-403.

George E, Lucas GM, Nadkarni GN, Fine DM, Moore R, Atta MG 2010. Kidney function and the risk of cardiovascular events in HIV-1 infected patients. AIDS 24: 387-394.

Ras J, Blaxhult A, Sundelin B 2000. Sudden unexpected death as a consequence of indinavir-induced nephropathy. A case report. APMIS 108: 581-583.

Ronchini KROM, Goto H, Duarte AJS, Gidlund M 2013. Anti-oxidized LDL antibodies as atherosclerosis development markers in HIV patients undergoing antiretroviral therapy: a longitudinal cohort study. Int Trends Immun 1: 73-79.

Singh M, Arora R, Jawas E 2010. HIV protease inhibitors induced prolongation of the QT interval: electrophysiology and clinical implications. Am J Ther 17: e193-e201.

Soares SRC, Carvalho-Vieira R, Ramos-Sanchez E, Catanozi S, da Silva LFF, Mauad T, Gidlund M, Goto H, Garcia ML 2009. Air pollution and antibodies against modified lipoproteins are associated with atherosclerosis and vascular remodeling in hyperlipemic mice. Atherosclerosis 207: 368-373.

Ugawa T, Kakuta H, Moritani H, Shikama H 2002. Experimental model of escape phenomenon in hamsters and the effectiveness of YM-53601 in the model. Br J Pharmacol 135: 1572-1578.

Worm SW, Kamara DA, Reiss P, Fontas E, de Wit S, El-Sadr W, Monforte AD, Law M, Philips A, Ryom L, Dabis F, Weder R, Sabin C, Lundgren D, D:A:D Study Group 2012. Evaluation of HIV protease inhibitor use and the risk of sudden death or nonhemorrhagic stroke. J Infect Dis 205: 535-539. 